\title{
The Graph of Annihilating Ideals
}

\author{
Nazar H. Shuker
}

Fryad H. Abdulqadir

nazarh_2013@yahoo.com

College of Computer Science and Mathematics

University of Mosul, Mosul, Iraq

Received on: 17/11/2013

Accepted on: 12/02/2014

\section{ABSTRACT}

Let $\mathrm{R}$ be a commutative ring with identity and $A G(R)$ be the set of ideals with non-zero annihilators. The annihilating ideal graph $A G(R)$ is a graph of vertex set $A G(R) \backslash\{(0)\}$ and two distinct ideal vertices $I$ and $J$ are adjacent if and only if $I J=$ (0). In this paper, we establish a new fundamental properties of $A G(R)$ as well as its connection with $\Gamma(\mathrm{R})$.

Keywords: Annihilating ideal graph, zero divisor graph, reduced rings, finite local rings, rings integer modulo $\mathrm{n}$

\section{بيان للمثاليات التالفة}

$$
\text { فرباد عبد القادر }
$$

$$
\text { جلية علوم الحاسوب واليراضيات الموصل، الدوصل، العراق }
$$

تاريخ قبول البحث: 2014/02/12

تاريخ استلام البحث: 2013/11/17

\section{الملخص}

لتكن R حلقة إبدالية تحتوي على العنصر المحايد، وان AG(R) مجموعة المثاليات ذات تالف غير

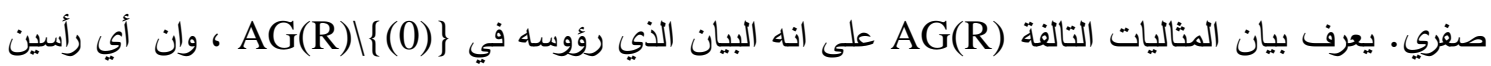

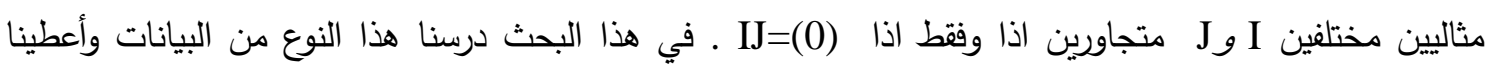

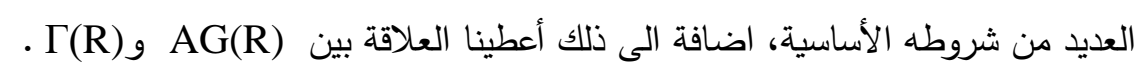

الكلمات المفتاحية: بيان تالف المثاليات، بيان قاسم الصفر ، الحلقات المختزلة، الحلقات المنتهية المحلية، الحلقات

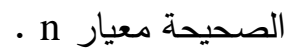

\section{Introduction:}

Let $\mathrm{R}$ be a commutative ring with identity, and let $\mathrm{Z}(\mathrm{R})$ be its set of zero divisors. We associate a simple graph $\Gamma(\mathrm{R})$ to $\mathrm{R}$ with vertices $\mathrm{Z}^{*}(\mathrm{R})=\mathrm{Z}(\mathrm{R}) \backslash\{(0)\}$, the set of all non-zero zero divisors of $\mathrm{R}$, and for distinct $x, y \in \mathrm{Z}^{*}(\mathrm{R})$, the vertices $\mathrm{x}$ and $\mathrm{y}$ are adjacent if and only if $x y=0$. Thus,$\Gamma(R)$ is empty graph iff $R$ is an integral domain.

Beck introduced the concept of zero divisor graph of a commutative ring in [4]. In the recent years zero divisor graph have been extensively studied by many authors in $[1,2,3,8]$.

An ideal I of $\mathrm{R}$ is said to be annihilating ideal if there exists a non-trivial ideal $\mathrm{J}$ of $\mathrm{R}$ such that $\mathrm{I} J=(0)$. Let $\mathrm{AG}(\mathrm{R})$ be the set of annihilating ideals of $\mathrm{R}$. The annihilating 
ideal graph $A G(R)$ is a graph with vertex set $A G^{*}(R)=A G(R) \backslash\{(0)\}$ such that there is an edge between vertices $I$ and $J$ if and only if $I \neq J$ and $I J=(0)$. The idea of annihilating ideal graph was introduced by Behboodi and Rakeei in [5] and [6].

In the present paper, we investigate the annihilating ideal graph $A G(R)$. We establish a new of its basic properties and its relation of $\Gamma(R)$.

\section{Recall that:}

1. $\mathrm{R}$ is called reduced if $\mathrm{R}$ has no non-zero nilpotent element.

2.The distance $d(u, v)$ between two vertices $u$ and $v$ of a connected graph $\Gamma$ is the minimum of the lengths of the $u-v$ paths of $\Gamma$ [7].

3. The degree of the vertex $a$ in the graph $\Gamma$ is the number of edges of $\Gamma$ incident with $a$ [7].

4. The graph $\Gamma$ is called a plane graph if it can be drawn in the plane with their edges crossing. A graph which is an isomorphic to a plane graph is called a planer graph[7].

5. A graph $\Gamma$ is bipartite graph, if it is possible to partition the vertex set of $\Gamma$ into two subsets $V_{1}$ and $V_{2}$ such that every element of edges of $\Gamma$ joins a vertex of $V_{1}$ to a vertex of $V_{2}$. A complete bipartite graph with partite sets $V_{1}$ and $V_{2}$ where, $\left|V_{1}\right|=m$ and $\left|V_{2}\right|=\mathrm{n}$, is then denoted by $\mathrm{K}_{\mathrm{m}, \mathrm{n}}[7]$.

\section{Annihilating ideal graph:}

In this section, we consider annihilating ideal graph, we give some of its basic properties and provide some examples.

Definition2.1[5]: Let $\mathrm{R}$ be a ring and let $\mathrm{I}$ and $\mathrm{J}$ are distinct non-trivial ideals of R.Then , $I$ and $J$ are adjacent ideal vertices in $A G(R)$ if $I J=(0)$.

From now on, we shall use the symbol I-J to denote for two adjacent ideal vertices I and $\mathrm{J}$. We start this section with the following example .

Example1: Let $Z_{24}$ be the ring of integers modulo 24 . The graph $A G\left(Z_{24}\right)$ can be drawn as follows:

(3)

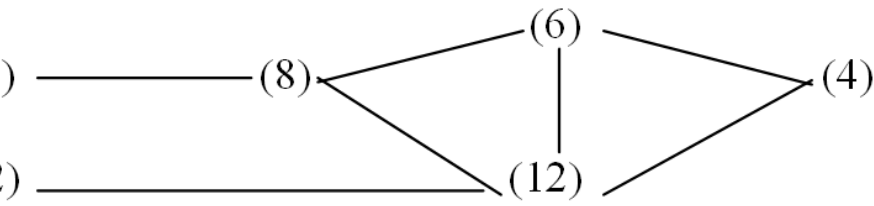

The following result is an easy consequence of definition of 2.1 .

Lemma2.2: If $I$ and $J$ are non-trivial ideals of $\mathrm{R}$ such that $I \cap J=(0)$, then $\mathrm{I}-\mathrm{J}$ is an edge of $\mathrm{AG}(\mathrm{R})$ and $I \cup J \subseteq \mathrm{Z}(\mathrm{R})$.

The converse of Lemma2.2 is not true in general, as the following example shows.

Example2: Let $Z_{12}$ be the ring of integers modulo 12.Then , (2) - (6) is an edge of the graph $\mathrm{AG}\left(\mathrm{Z}_{12}\right)$, but $(2) \cap(6) \neq(0)$.

We now give a sufficient condition for the converse of Lemma2.2 to be true.

Proposition 2.3: Let $\mathrm{R}$ be a reduced ring, and let $\mathrm{I}-\mathrm{J}$ be an edge in $A G(\mathrm{R})$. Then , $I \cap J=(0)$. 
Proof: Let $a \in I \cap J$. Then , $a \in I$ and $a \in J$, this implies that $a^{2} \in I J=(0)$, so $a^{2}=$ 0 . Since, $\mathrm{R}$ is a reduced ring, then $a=0$. Therefore, $I \cap J=(0)$.

The next result illustrates that the distance of any two nilpotent ideal vertices of $\mathrm{AG}(\mathrm{R})$ is at most 2 .

Theorem2.4: Let I and J be two ideal vertices of AG(R). If either I or J is a nilpotent, then $\mathrm{d}(\mathrm{I}, \mathrm{J}) \leq 2$.

Proof: Let $d(I, J)=3$. Then, there is a path from $I$ to $J$ in $A G(R)$ say $I-K-L-J$. Let $I$ be a nilpotent ideal of $R$. Then, there exists an integer $n>1$ such that $\mathrm{I}^{\mathrm{n}}=(0)$. Consider the sequence $\mathrm{L}, \mathrm{LI}, \mathrm{LI}^{2}, \ldots, \mathrm{LI}^{\mathrm{n}}$. Let $\mathrm{m}$ be the smallest integer in which $\mathrm{LI}^{\mathrm{m}} \neq(0)$. Hence, $\mathrm{LI}^{\mathrm{m}+1}=(0)$. Obviously, $\mathrm{LI}^{\mathrm{m}}$ adjacent to both $\mathrm{I}$ and $\mathrm{J}$. This contradict the fact that $\mathrm{d}(\mathrm{I}, \mathrm{J})=3$. Therefore, $\mathrm{d}(\mathrm{I}, \mathrm{J}) \leq 2$.

The next result illustrates the degree of a vertex adjacent to the set of zero divisors of $\mathrm{R}$.

Proposition 2.5: Let $R$ be a finite ring and let $Z(R)$ be an ideal of $R$. If $I-Z(R)$ is an edge in $\mathrm{AG}(\mathrm{R})$, then $\operatorname{deg}(\mathrm{I})=|\mathrm{AG}(R)|-1$.

Proof: Suppose that $I-Z(R)$ be an edge in $A G(R)$, it follows that $I \cdot Z(R)=(0)$. Let $J$ be any vertex of $\mathrm{AG}(R)$.Then, by Lemma2.2, $\mathrm{J}$ is a subset of $\mathrm{Z}(\mathrm{R})$. This implies that $\mathrm{I} \cdot \mathrm{J}=(0)$.Thus, $\mathrm{I}$ is adjacent to all vertices of $\mathrm{AG}(R)$. This means that $\operatorname{deg}(\mathrm{I})=|\mathrm{AG}(R)|$ 1.

Example3: Let $\mathrm{Z}_{16}$ be the ring of integers modulo 16. The vertices of $A G\left(Z_{16}\right)$ are $\mathrm{I}=(8)$

, J=(4) and $\mathrm{K}=(2)=\mathrm{Z}\left(\mathrm{Z}_{16}\right)$. Clearly $\operatorname{deg}(\mathrm{I})=\operatorname{deg}(\mathrm{J})=\left|\mathrm{AG}\left(Z_{16}\right)\right|=3-1$.

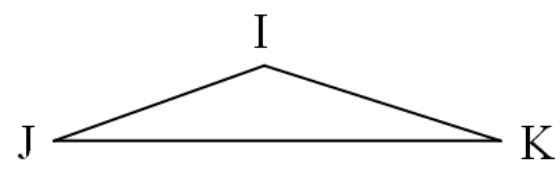

The next result considers the adjacency of two minimal ideals in the graph AG(R).

Proposition 2.6: Every two distinct minimal ideals of $\mathrm{R}$ are adjacent in $\mathrm{AG}(R)$.

Proof: Let $\mathrm{M}$ and $\mathrm{N}$ be two distinct minimal ideals of R. Since, $\mathrm{M}$ and $\mathrm{N}$ contain $\mathrm{MN}$, then $\mathrm{MN}=\mathrm{M}=\mathrm{N}$ or $\mathrm{MN}=(0)$. The first case is not true because $\mathrm{M}$ and $\mathrm{N}$ are distinct ideals. Thus , $\mathrm{MN}=(0)$. This means that $\mathrm{M}$ and $\mathrm{N}$ are adjacent vertices in $\mathrm{AG}(R)$.

Example4: Let $\mathrm{Z}_{18}$ be the ring of integers modulo 18 .

(3)

(9)

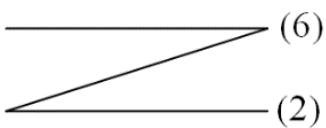

Clearly, the minimal ideals of $Z_{18}$ are (6) and (9), which are adjacent vertices in $\mathrm{AG}\left(\mathrm{Z}_{18}\right)$.

The next result considers the number of minimal ideals of $\mathrm{R}$.

Theorem2.7: If $A G(R)$ is a planar graph, then $\mathrm{R}$ has at most four minimal ideals.

Proof: Suppose that $R$ has five minimal ideals say $M_{1}, M_{2}, M_{3}, M_{4}$ and $M_{5}$. By Proposition2.6, any two of $\mathrm{M}_{1}, \mathrm{M}_{2}, \mathrm{M}_{3}, \mathrm{M}_{4}$ and $\mathrm{M}_{5}$ are adjacent. This means that 
$A G(R)$ contains the complete graph $\mathrm{K}_{5}$. This is contradiction that $A G(R)$ is a planar graph (See the Kuratowsky Theorem in [7]). Therefore, $\mathrm{R}$ has at most four minimal ideals.

Example5: Let $\mathrm{Z}_{16}$ be the ring of integers modulo 16 .

Clearly, the graph $A G\left(Z_{16}\right)$ is a planar graph and the

(2)

$(4)$ $(8)$ only minimal ideal of $Z_{16}$ is (8).

\section{The graphs $\Gamma(R)$ and $A G(R)$}

In this section, we consider the relationship between $\Gamma(R)$ and $A G(\mathrm{R})$.

It is natural to ask whether $\Gamma(R)$ and $A G(\mathrm{R})$ are isomorphic, the answer is negative, as the following example shows.

Example6: Let $\mathrm{Z}_{12}$ be the ring of integer modulo 12. Then, the number of vertices of $\Gamma\left(\mathrm{Z}_{12}\right)$ is 7 , while the number of vertices of $A G\left(\mathrm{Z}_{12}\right)$ is 4 . Obviously, $\Gamma\left(\mathrm{Z}_{12}\right)$ and $A G\left(\mathrm{Z}_{12}\right)$ are not isomorphic.

The next result explores the relation between the set of zero divisors of $\mathrm{R}$ and the vertices of $A G(R)$.

Theorem3.1: For any ring $\mathrm{R}, \mathrm{Z}(\mathrm{R})=\mathrm{U}\{I: I$ is an ideal vertex of $\mathrm{AG}(R)\}$.

Proof: Let $0 \neq \mathrm{x} \in Z(R)$. Then, there exists $\mathrm{y} \in Z^{*}(R)$ such that $\mathrm{xy}=0$.This implies that $(\mathrm{x})(\mathrm{y})=(0)$. If $(\mathrm{x})=\mathrm{R}$, then $\mathrm{x}$ is a unit element. This contradicts the fact that $\mathrm{x} \in Z^{*}(R)$. So, $(\mathrm{x}) \neq \mathrm{R}$. Since $(\mathrm{x}) \quad$ is adjacent to $(\mathrm{y})$, then $\mathrm{x} \in(x) \in$ $\{I: I$ is an ideal vertex of $\mathrm{AG}(R)\}$. Therefore, $\mathrm{x} \in \mathrm{U}\{I: I$ is an ideal vertex of $\mathrm{AG}(R)\}$.

Conversely, suppose that $x \in \cup\{I: I$ is an ideal vertex of $A G(R)\}$. Then, $x \in I$ for some vertex $\mathrm{I}$ of $\mathrm{AG}(R)$. By Lemma2.2, $\mathrm{x} \in Z(R)$.Hence, $\mathrm{Z}(\mathrm{R})=\mathrm{U}\{I: I$ is a vertex of $\mathrm{AG}(R)\}$.

Let us give the following easy result.

Proposition3.2: Let $\Gamma(\mathrm{R})$ and $\mathrm{AG}(R)$ are finite graphs, then $|\Gamma(R)| \geq|\mathrm{AG}(R)|$.

The following result demonstrates the isomorphism between $\Gamma(R)$ and AG(R) by considering $\mathrm{R}=\mathrm{Z}_{\mathrm{n}}$.

Theorem3.3: Let $n>1$ be a non-prime integer. Then, $\Gamma\left(Z_{n}\right)$ contains a subgraph which isomorphic with $\mathrm{AG}\left(\mathrm{Z}_{\mathrm{n}}\right)$.

Proof: Define the graph $\mathrm{G}$ by $\mathrm{G}=\left\{a-b: a-b\right.$ is an edge in $\Gamma\left(\mathrm{Z}_{\mathrm{n}}\right), a|\mathrm{n}, \mathrm{b}| \mathrm{n}$ and $a \neq b$. Obviously, $\mathrm{G}$ is a subgraph of $\Gamma\left(\mathrm{Z}_{\mathrm{n}}\right)$. Now, define a function $\mathrm{f}: \mathrm{G} \rightarrow \mathrm{AG}\left(\mathrm{Z}_{\mathrm{n}}\right)$ by $\mathrm{f}(a)=(a)$, with $a \in \mathrm{G}$. Clearly $\mathrm{f}$ is onto. Now, for any distinct vertices $a, \mathrm{~b} \in \mathrm{G}, a \mid \mathrm{n}$ and $\mathrm{b} \mid \mathrm{n}$. So, $\mathrm{f}(a)=(a) \neq(\mathrm{b})=\mathrm{f}(\mathrm{b})$. Thus, $\mathrm{f}$ is one to one. Now, suppose that $a-b$ is an edge in G. Then, $a b=0$, so $(a)(b)=(0)$. This shows that $f(a) f(b)=(0)$, and hence $\mathrm{f}(a)-\mathrm{f}(\mathrm{b})$ is an edge in $\mathrm{AG}\left(\mathrm{Z}_{\mathrm{n}}\right)$. Thus $\mathrm{f}$ preserves the adjacency property. This proves that $\mathrm{G} \cong \mathrm{AG}\left(\mathrm{Z}_{\mathrm{n}}\right)$.

The following result gives a sufficient conditions for two vertices of $\Gamma(\mathrm{R})$ such that their annihilators are adjacent ideal vertices in $\mathrm{AG}(R)$.

Theorem3.4: If $a$ and $\mathrm{b}$ are two vertices in $\Gamma(\mathrm{R})$ such that $\mathrm{d}(a, \mathrm{~b})=3$, then $\operatorname{Ann}(a)$ and $\mathrm{Ann}(\mathrm{b})$ are adjacent ideal vertices in $\mathrm{AG}(R)$. 
Proof: Since, $a, b \in Z^{*}(R)$, then both $\operatorname{Ann}(a)$ and Ann(b) are non-zero. On the other hand, $\mathrm{d}(a, \mathrm{~b})=3$. This means that neither $\mathrm{b} \in \operatorname{Ann}(a)$ nor $a \in \operatorname{Ann}(\mathrm{b})$. Then, neither $\operatorname{Ann}(a)=\mathrm{R}$ nor $\operatorname{Ann}(\mathrm{b})=\mathrm{R}$. So, both $\operatorname{Ann}(a)$ and $\operatorname{Ann}(\mathrm{b})$ are nontrivial ideals. If we assume that $\operatorname{Ann}(a) \operatorname{Ann}(b) \neq(0)$, then there exists $c \in \operatorname{Ann}(a)$ and $\mathrm{d} \in \operatorname{Ann}(b)$ such that $\mathrm{cd} \neq 0$. Clearly $a(\mathrm{~cd})=\mathrm{b}(\mathrm{cd})=0$. This means that $a-c d-b$ is a path in $\Gamma(\mathrm{R})$. This contradicts the fact that $\mathrm{d}(a, \mathrm{~b})=3$. Therefore, $\operatorname{Ann}(a)$ and $\operatorname{Ann}(\mathrm{b})$ are adjacent ideal vertices in $\mathrm{AG}(R)$.

Example7: Let $Z_{12}$ be the ring of integers modulo 12. Clearly, $d((3),(10))=3$ in $A G\left(Z_{12}\right)$ and $A n n(3) A n n(10)=(4)(6)=(0)$. This means that $A n n(3)$ and $A n n(10)$ are adjacent in $\mathrm{AG}\left(\mathrm{Z}_{12}\right)$.

We end this paper by showing that,

Proposition3.5: If $\mathrm{R}$ is a finite local ring, then $A G(R) \neq K_{m n}$ for any integers $\mathrm{m}, \mathrm{n}>1$. Proof: Suppose that $A G(R)=K_{m n}$ for some integers $\mathrm{m}, \mathrm{n}>1$, and let $\mathrm{A}=\left\{\mathrm{I}_{1}, \mathrm{I}_{2}, \ldots, \mathrm{I}_{\mathrm{n}}\right\}$ and $\mathrm{B}=\left\{\mathrm{J}_{1}, \mathrm{~J}_{2}, \ldots, \mathrm{J}_{\mathrm{m}}\right\}$ be the partition of $A G(R)$. Since, $\mathrm{R}$ is a local ring, then by Theorem1.2 in [9], $\mathrm{Z}(\mathrm{R})$ is an ideal of $\mathrm{R}$ and there exists a vertex $a$ of $\Gamma(R)$ such that $a \cdot \mathrm{Z}(\mathrm{R})=(0)$. It follows that $(a) \cdot \mathrm{Z}(\mathrm{R})=(0)$. Hence, $\mathrm{Z}(\mathrm{R})$ is a vertex of $A G(R)$, yielding $Z(R) \in A$ or $(R) \in B$. Now, if $(R) \in A$, then $\mathrm{J}_{\mathrm{i}} \mathrm{Z}(\mathrm{R})=(0)$ for all $\mathrm{i}=1,2, \ldots, \mathrm{m}$. By Theorem3.1, $\mathrm{J}_{\mathrm{i}} \mathrm{J}_{\mathrm{k}}=(0)$ for $\mathrm{i} \neq \mathrm{k}$. This contradicts the fact that $\mathrm{J}_{\mathrm{i}}$ and $\mathrm{J}_{\mathrm{k}}$ are not adjacent. If $(R) \in B$, this will lead to a contradiction. Thus, $\mathrm{AG}(\mathrm{R}) \neq \mathrm{K}_{\mathrm{mn}}$ for any integers $\mathrm{m}, \mathrm{n}>1$. 


\section{REFERENCES}

[1] S. Akbari , H. R. Maimani and S. Yassemi, " when zero divisor graph is planner or a complete r-partite graph", J. Algebra 270 (2003), 1 , 169-180.

[2] D. F. Anderson , A. Frazier , A, Laune and P. S. Livingston "The zero divisor graph of a commutative ring $\Pi$ Lecture note in pure and applied Mathematics" , 220 , Dekker, Newyork (2001).

[3] D. F. Anderson and P. S. Livingston, "The zero divisor graph of a commutative ring", J. Algebra. 217 (1999), 434-447.

[4] I. Beck, "Coloring of commutative ring" , J. Algebra 116 (1988) , 1, 206-226.

[5] M. Behboodi and Z. Rakeei, "The Annihilating-Ideal Graph of Commutative Rings I", J. Algebra Appl. 10 (2011), 741-753.

[6] M. Behboodi and Z. Rakeei, "The Annihilating-Ideal Graph of Commutative Rings II" , J. Algebra Appl. 10 (2011), 727-739.

[7] C. Gary and L. Linda , "Graphs \& Diagraphs", California, A divison of Wadsworth Inc(1986).

[8] R. Levy and J. Shapiro, "The zero divisor graph of nonneam regular rings", comm. Algebra 30 (2002), 2 , 745-750.

[9] A. Michael , S. Joe and T. Wallace, "Zero divisor ideals and realizable zero divisor graphs", Communicated by Scott Chapman (2009). 